\title{
Strange Electric Form Factor of the Proton
}

\author{
D. B. Leinweber, ${ }^{1}$ S. Boinepalli, ${ }^{1}$ A. W. Thomas,,${ }^{1,2}$ P. Wang, ${ }^{1}$ A. G. Williams, ${ }^{1}$ \\ R. D. Young, ${ }^{1,2}$ J. M. Zanotti, ${ }^{1,3}$ and J. B. Zhang ${ }^{1}$ \\ ${ }^{1}$ Special Research Centre for the Subatomic Structure of Matter, and Department of Physics, University of Adelaide, \\ Adelaide SA 5005, Australia \\ ${ }^{2}$ Jefferson Laboratory, 12000 Jefferson Avenue, Newport News, Virginia 23606 USA \\ ${ }^{3}$ School of Physics, University of Edinburgh, Edinburgh EH9 3JZ, United Kingdom
}

(Received 20 January 2006; published 10 July 2006)

\begin{abstract}
By combining the constraints of charge symmetry with new chiral extrapolation techniques and recent low-mass quenched lattice QCD simulations of the individual quark contributions to the electric charge radii of the baryon octet, we obtain an accurate determination of the strange electric charge radius of the proton. While this analysis provides a value for $G_{E}^{s}\left(Q^{2}=0.1 \mathrm{GeV}^{2}\right)$ in agreement with the best current data, the theoretical error is comparable with that expected from future HAPPEX results from JLab. Together with the earlier determination of $G_{M}^{s}$, this result considerably constrains the role of hidden flavor in the structure of the nucleon.
\end{abstract}

DOI: 10.1103/PhysRevLett.97.022001

PACS numbers: $12.38 . \mathrm{Gc}, 12.39 . \mathrm{Fe}, 13.40 . \mathrm{Em}, 14.20 . \mathrm{Dh}$

One of the great challenges of modern hadron physics is to unravel the precise role of hidden flavors in the structure of the nucleon. Because of their relatively light mass, strange quarks are expected to play the biggest role, and it is with respect to strangeness that there has recently been enormous experimental progress. Indeed, new results on strangeness in the nucleon have been reported recently from the HAPPEX [1] and G0 [2] Collaborations at JLab, which complement earlier work at MIT-Bates [3] and Mainz [4]. The situation is by far the best at $Q^{2}=$ $0.1 \mathrm{GeV}^{2}$, where, in addition to the usual linear combination of electric and magnetic form factors, a measurement of parity violation on ${ }^{4} \mathrm{He}$ allowed an accurate extraction of $G_{E}^{s}$, namely, $G_{E}^{s}\left(Q^{2}=0.1 \mathrm{GeV}^{2}\right)=-0.013 \pm 0.028$. A new experimental investigation, in which this error is expected to be reduced by roughly a factor of 2 , was conducted in late 2005. This makes it imperative to find, as we report, a way to make a theoretical estimate of comparable accuracy - or better.

With respect to the strange magnetic form factor of the proton, we recently reported a calculation an order of magnitude more precise than current experiments $[5,6]$. This calculation exploited the advances in lattice QCD which have enabled quenched QCD (QQCD) simulations of magnetic moments at pion masses as low as $0.3-$ $0.4 \mathrm{GeV}$ [7-9], as well as the development of new chiral extrapolation techniques $[10,11]$. To minimize theoretical uncertainty, an essential input was the precise (experimental) measurements of the magnetic moments of the ground state hyperons - a luxury unfortunately not available for charge radii. Nevertheless, we show here that even the limited data on hyperon charge radii, when combined with new lattice simulations and chiral extrapolation techniques, yield a precision commensurate with the published data. Alternatively, using the best estimates for the charge radii of the valence $u$ and $d$ quarks, extracted from the lattice simulations, yields a determination of the strangequark contribution to the proton charge radius with an uncertainty comparable with that anticipated from the latest HAPPEX measurement.

As illustrated in Fig. 1, the three point function required to extract an electromagnetic form factor in lattice QCD involves two topologically distinct processes (each incorporating an arbitrary number of gluons and quark loops). The left-hand diagram illustrates the connected insertion of the current to one of the "valence" quarks of the baryon. In the right-hand diagram, the external field couples to a quark loop. The latter process, where the loop involves an $s$ quark, is entirely responsible for $G_{E}^{s}$.

Charge symmetry, which is the invariance of the QCD Hamiltonian under rotations by $180^{\circ}$ about the 2 -axis in isospace, is typically respected at a level better than $1 \%$ in nuclear physics [12]. This gives one confidence in using it to extract the strange form factors experimentally and we shall also use it. Under charge symmetry, the electric or magnetic form factors of the octet baryons satisfy [13]:

$$
\begin{array}{cc}
p=e_{u} u^{p}+e_{d} d^{p}+O_{N}, \quad n=e_{d} u^{p}+e_{u} d^{p}+O_{N}, \\
\Sigma^{+}=e_{u} u^{\Sigma}+e_{s} s^{\Sigma}+O_{\Sigma}, & \Sigma^{-}=e_{d} u^{\Sigma}+e_{s} s^{\Sigma}+O_{\Sigma} .
\end{array}
$$

Here $p, \Sigma^{-}$, etc., represent any form factor of the physical proton and $\Sigma^{-}$and similarly for the other baryons. For the
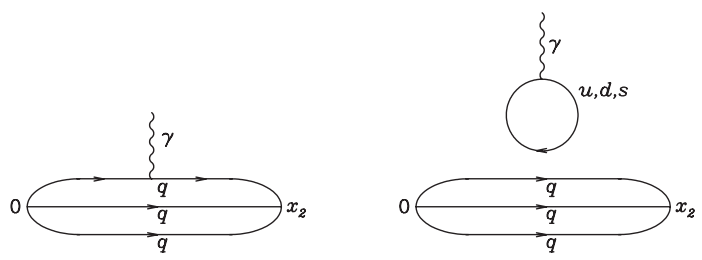

FIG. 1. Diagrams illustrating the two topologically different insertions of the current within the framework of lattice QCD. 
present application, we take them to be the mean-square charge radii, defined as $-6 d /\left.d Q^{2} G_{E}\left(Q^{2}\right)\right|_{Q^{2}=0}$. Similarly, $u^{p}$ denotes the contribution to the proton mean-square charge radius from the two valence $u$ quarks (for $u$ quarks of unit charge); see the left-hand side of Fig. 1.

Of course, the strange-quark contribution is entirely contained in the quark loops $O_{N}$, illustrated in the righthand side of Fig. 1. However, one has to separate the contributions associated with $u$ and $d$ quarks from those involving $s$ quarks. To this end, we define ${ }^{\ell} u,{ }^{\ell} d$, and ${ }^{\ell} s$ as the mean-square charge radii of the loop contributions associated with $u, d$, and $s$ quarks (of unit charge), respectively. Hence, we may write:

$$
O_{N}=\frac{2}{3} \ell u-\frac{1}{3}{ }^{\ell} d-\frac{1}{3}{ }^{\ell} s=\frac{{ }^{\ell} s}{3}\left(\frac{1-{ }^{\ell} R_{d}^{s}}{{ }^{\ell} R_{d}^{s}}\right),
$$

where the ratio of $s$ - and $d$-quark loops ${ }^{\ell} R_{d}^{s} \equiv{ }^{\ell} s /{ }^{\ell} d$ is expected to lie in the range $(0,1)$. Note that, in deriving Eq. (2), we have used charge symmetry (or $m_{u}=m_{d}$ ) to set ${ }^{\ell} u={ }^{\ell} d$ [13]. Since the chiral coefficients for the $d$ and $s$ loops in the right-hand side of Fig. 1 are identical, the main difference comes from the mass of the $K$ compared with that of the $\pi$.

With a little algebra, $O_{N}$, and hence ${ }^{\ell} s$, may be isolated from Eqs. (1) and (2):

$$
{ }^{\ell} s=\left(\frac{{ }^{\ell} R_{d}^{s}}{1-{ }^{\ell} R_{d}^{s}}\right)\left[2 p+n-\frac{u^{p}}{u^{\Sigma}}\left(\Sigma^{+}-\Sigma^{-}\right)\right] .
$$

It is worth emphasizing that this expression is an exact consequence of QCD, under the assumption of charge symmetry.

There is a relation similar to Eq. (3) involving the charge radii of the $\Xi$ baryons and the ratio of the charge radii of $u$ quarks in the $n$ and $\Xi^{0}$, but as there are no experimental data at all for the cascades we do not show it. Even in the case of the $\Sigma$ hyperons, there are data only for the $\Sigma^{-}-$ from the SELEX Collaboration at Fermilab [14]. Contrasting the magnetic moments, the experimental error is relatively large, $\Sigma^{-}=-0.61 \pm 0.12 \pm 0.09 \mathrm{fm}^{2}$. Nevertheless, as we shall see, even this is enough, in combination with lattice QCD estimates of the ratio of charge radii in the $\Sigma^{+}$compared with the $\Sigma^{-}$, to obtain an accurate estimate of the strangeness radius.

Combining the experimentally measured mean-square charge radii of the $p$ and $n(0.757 \pm 0.014$ and $-0.116 \pm$ $0.002 \mathrm{fm}^{2}$, respectively [15]), we find:

$$
\begin{aligned}
\ell_{s}= & \left(\frac{{ }^{\ell} R_{d}^{s}}{1-{ }^{\ell} R_{d}^{s}}\right)\left[1.398-\frac{u^{p}}{u^{\Sigma}}\left(1+\left|\frac{\Sigma^{+}}{\Sigma^{-}}\right|\right) 0.61\right. \\
& \pm 0.12 \pm 0.09] .
\end{aligned}
$$

To obtain $O_{N}$, the necessary input from lattice QCD is now the two ratios in Eq. (4), namely, $u^{p} / u^{\Sigma}$ and $\Sigma^{+} / \Sigma^{-}$. Figures $2-5$ show the state of the art calculations from

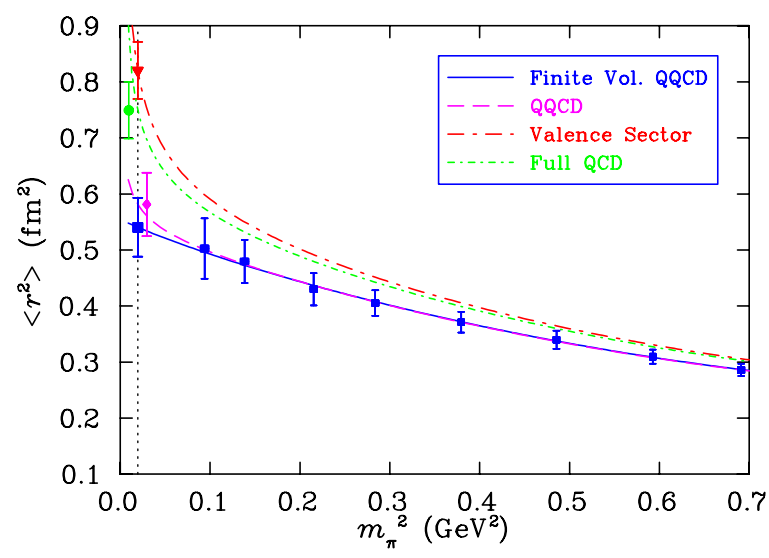

FIG. 2 (color online). Mean-square charge radius of the $\Sigma^{+}$, as a function of the pion mass squared, calculated in quenched lattice QCD [27]. The solid curve displays the finite volume fit, the infinite-volume limit of quenched QCD is shown by the dashed curve, the valence sector of full QCD is represented by the long-dashed-dotted curve, and the short-dashed-dotted curve shows the total result, including the disconnected loops.

which we can extract these ratios. The numerical simulations of the electromagnetic form factors presented here are carried out using the fat link irrelevant clover (FLIC) fermion action [7], in which the irrelevant operators, introduced to remove fermion doublers and lattice spacing artifacts, are constructed with APE smeared links [16]. Perturbative renormalizations are small for smeared links, and the mean-field improved coefficients used here are sufficient to remove $\mathcal{O}(a)$ errors from the lattice fermion action [17].

The $\mathcal{O}(a)$-improved conserved vector current [18] is used. Nonperturbative improvement is achieved via the FLIC procedure, where the terms of the Noether current having their origin in the irrelevant operators of the fermion action are constructed with mean-field improved

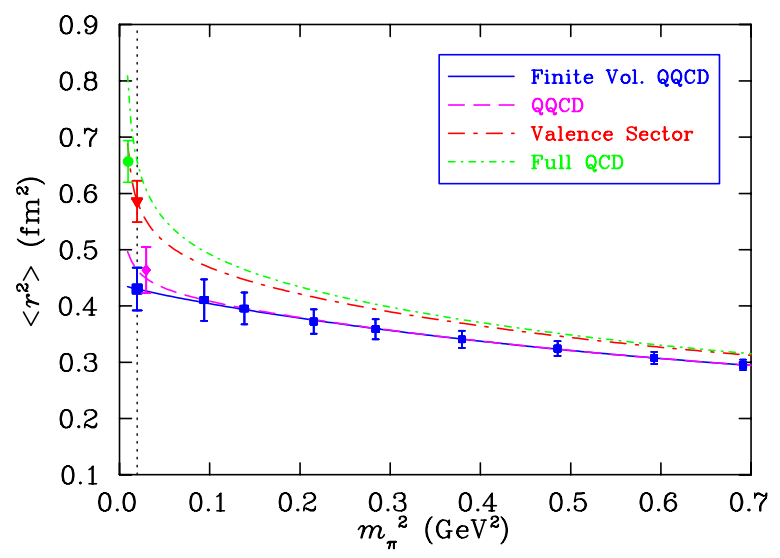

FIG. 3 (color online). Absolute value of the mean-square charge radius of the $\Sigma^{-}$, as a function of pion mass squared, calculated in quenched lattice QCD [27]. The curve descriptions are the same as those in Fig. 2. 


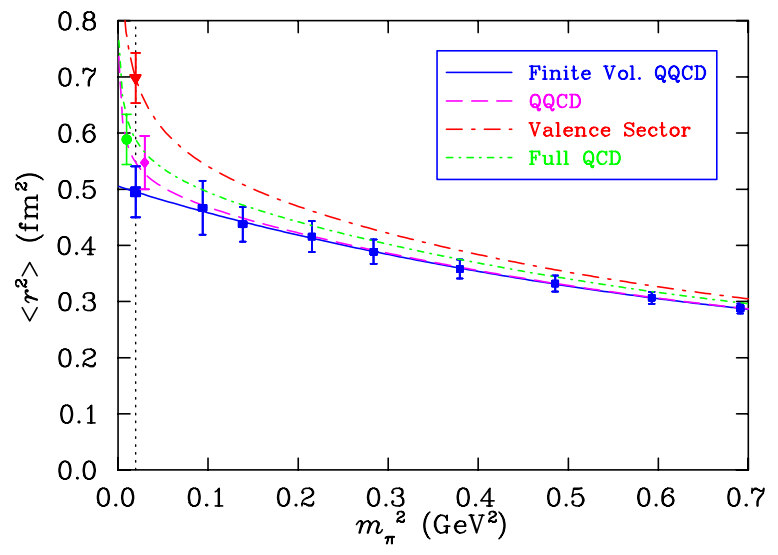

FIG. 4 (color online). Mean-square charge radius of a single $u$ quark (of unit charge) in the proton, calculated in quenched lattice QCD [27]. The curve descriptions are the same as those in Fig. 2.

APE smeared links. The results presented here are obtained using established techniques [19] from a sample of 400, $20^{3} \times 40$ mean-field $\mathcal{O}\left(a^{2}\right)$-improved Luscher-Weisz [20] gauge field configurations, having a lattice spacing of $0.128 \mathrm{fm}$, determined by the Sommer scale $r_{0}=0.50 \mathrm{fm}$. Charge radii are obtained from dipole fits to quark-sector electric form factors, calculated at $Q^{2}=0.227 \pm$ $0.002 \mathrm{GeV}^{2}$. The dipole form is well supported physically and in lattice QCD simulations over a range of quark masses [21].

From Figs. 2 and 3, we see that the radius of the $\Sigma^{+}$ grows significantly larger than that of the $\Sigma^{-}$as we approach the chiral limit. This has a relatively simple, physical interpretation in terms of the more extended spatial distribution of light quarks compared with strange. These simulation results are fit using finite-range regularized (FRR), quenched chiral effective field theory formulated on a finite volume. Upon extracting the fit, one can restore the infinite-volume limit within the effective field theory-

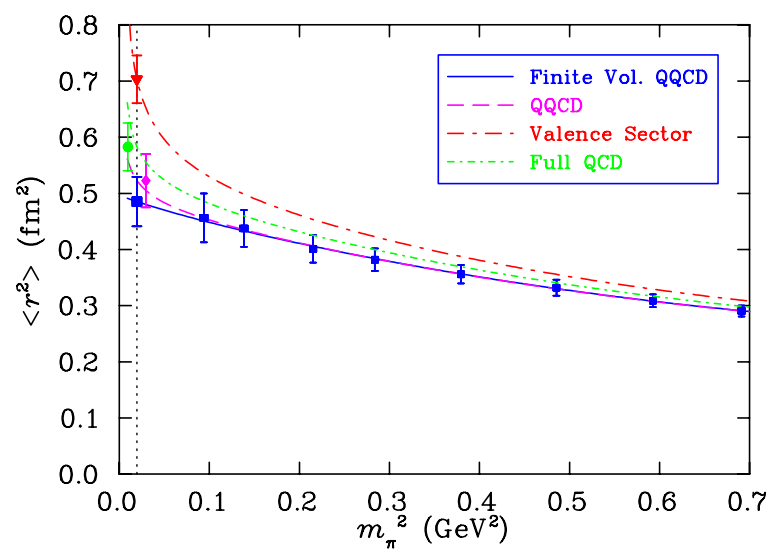

FIG. 5 (color online). Mean-square charge radius of a single $u$ quark (of unit charge) in the $\Sigma^{+}$, calculated in quenched lattice QCD [27]. The curve descriptions are the same as those in Fig. 2. where corrections are observed to be quite small right down to the lightest simulated pion mass. The QCD valence sector and total QCD (including loop insertions) contributions are then estimated by replacing the longrange "tail" of $\mathrm{Q} \chi \mathrm{PT}$ by the corresponding $\chi \mathrm{PT}$ tail in the same fashion as Refs. [5,11] — which extends upon the empirical success observed in Ref. [22].

Using the techniques described in Ref. [5], we estimate the effects of the dynamical sea and extrapolate the results to the physical quark mass. The ratio of the $\Sigma$ charge radii is found to be $\left|\Sigma^{+} / \Sigma^{-}\right|=1.141 \pm 0.036 \pm 0.010-$ where we observe the statistical fluctuations (first error) have been significantly reduced by constructing the ratio. The second uncertainty arises from the FRR scale dependence $\Lambda=0.8 \pm 0.1 \mathrm{GeV}$, constrained to provide agreement with experiment where available.

In Figs. 4 and 5, we show the corresponding analysis for the $u$-quark mean-square radii in the proton and $\Sigma^{+}$, respectively. Here we observe that there is very little sensitivity to the mass of the spectator quark, with the ratio of the extrapolated valence contributions giving our best estimate of $u^{p} / u^{\Sigma}=0.993 \pm 0.048 \pm 0.000$. The latter uncertainty indicates negligible sensitivity to the FRR scale. Putting these best estimates into Eq. (4), we obtain $\quad \ell_{s}={ }^{\ell} R_{d}^{s}\left(1-{ }^{\ell} R_{d}^{s}\right)^{-1}[0.11 \pm 0.06 \pm 0.02 \pm$ $\left.0.33 \mathrm{fm}^{2}\right]$, where the uncertainties are, respectively, statistical, systematic, and experimental.

In order to complete the evaluation, we also need to estimate the ratio of disconnected $s$ to light quark loops, ${ }^{\ell} R_{d}^{s}$. No attempt has yet been made to evaluate this ratio in an $a b$ initio lattice QCD simulation, although future work could attempt this by building on the work of Refs. [23,24]. We follow the same procedure as in Ref. [5] but now for the charge radii rather than the magnetic moments, using the relative strength of the FRR loop contributions. With the FRR scale varying over the generous range $0.8 \pm 0.2 \mathrm{GeV}$, this provides ${ }^{\ell} R_{d}^{s}=0.16 \pm 0.04$.

The final result for the proton strange, mean-square charge radius is then $\left\langle r^{2}\right\rangle_{s}^{p}=-{ }^{\ell} s / 3=-0.007 \pm 0.004 \pm$ $0.002 \pm 0.021 \mathrm{fm}^{2}$, where the uncertainties are statistical, FRR scale, and experimental in origin. Lattice scale determination uncertainties are negligible. At $Q^{2}=0.1 \mathrm{GeV}^{2}$, we expect the first term in the expansion of the electric form factor to dominate, $G_{E}\left(Q^{2}\right)=-Q^{2}\left\langle r^{2}\right\rangle / 6$. The strangeness electric form factor, conventionally defined without the charge factor, is then found to be

$$
G_{E}^{s}\left(0.1 \mathrm{GeV}^{2}\right)=-0.009 \pm 0.005 \pm 0.003 \pm 0.027 \text {. }
$$

This is consistent with the analysis of world data at $0.1 \mathrm{GeV}^{2}$, namely, $G_{E}^{s}\left(0.1 \mathrm{GeV}^{2}\right)=-0.013 \pm 0.028$. The error on this theory calculation is of similar precision to the experiment.

It is interesting that a combination of data from the SELEX Collaboration at Fermilab, together with the constraints of charge symmetry and modern lattice QCD, can 
TABLE I. Results $\left(\mathrm{fm}^{2}\right)$ are compared with experiment. Uncertainties are statistical and systematic, respectively. Note that $\left\langle r^{2}\right\rangle_{s}^{p}$ is reported including the strange-quark charge.

\begin{tabular}{ccc}
\hline \hline Quantity & This work & Experiment \\
\hline$\left\langle r^{2}\right\rangle^{p}$ & $+0.685 \pm 0.047 \pm 0.051$ & $+0.757 \pm 0.014$ \\
$\left\langle r^{2}\right\rangle^{n}$ & $-0.158 \pm 0.029 \pm 0.016$ & $-0.116 \pm 0.002$ \\
$\left\langle r^{2}\right\rangle^{\Sigma^{-}}$ & $-0.657 \pm 0.037 \pm 0.045$ & $-0.610 \pm 0.150$ \\
$\left\langle r^{2}\right\rangle_{s}^{p}$ & $+0.001 \pm 0.004 \pm 0.004$ & $-0.010 \pm 0.022$ \\
\hline \hline
\end{tabular}

provide a tight constraint on the strange electric form factor of the proton. For the present time, the errors are dominated by the experimental errors in the knowledge of the $\Sigma^{-}$charge radius. It would clearly be valuable to have new and more accurate measurements on both the $\Sigma^{-}$and $\Sigma^{+}$ hyperons. Data on the $\Xi^{-}$(and $\Xi^{0}$ ) would, as in the case of the strangeness magnetic moment, provide a valuable additional constraint.

However, noting that the uncertainty in our result is dominated by the lack of precision in the observed $\Sigma^{-}$ charge radius, we also consider a somewhat more ambitious approach. That is, we remove the dependence on the hyperons altogether and require only the observed nucleon charge radii and our best estimate for the quark-sector connected-current insertion, either $u^{p}$ or $d^{p}$,

$$
\begin{aligned}
{ }^{\ell} s & =\left(\frac{{ }^{\ell} R_{d}^{s}}{1-{ }^{\ell} R_{d}^{s}}\right)\left[2 p+n-u^{p}\right], \\
& =\left(\frac{{ }^{\ell} R_{d}^{s}}{1-{ }^{\ell} R_{d}^{s}}\right)\left[p+2 n-d^{p}\right] .
\end{aligned}
$$

In this case, uncertainties in the lattice scale determination $a=0.128(6) \mathrm{fm}[6]$ become significant. Our best extrapolation of the valence sector contributions to the meansquare charge radii in full QCD gives $u^{p}=1.396 \pm$ $0.090 \pm 0.046 \pm 0.086 \mathrm{fm}^{2}$ and $d^{p}=0.553 \pm 0.063 \pm$ $0.010 \pm 0.047 \mathrm{fm}^{2}$, where the uncertainties are statistical, FRR scale, and lattice scale in origin. These values provide two independent evaluations of the strangeness meansquare charge radius $\left\langle r^{2}\right\rangle_{s}^{p}=0.000 \pm 0.006 \pm 0.007 \mathrm{fm}^{2}$ and $\left\langle r^{2}\right\rangle_{s}^{p}=0.002 \pm 0.004 \pm 0.004 \mathrm{fm}^{2}$, where the first uncertainty is the combined statistical and experimental errors, and the second is the combined FRR and lattice scale uncertainty. Combining these two in the extraction of the form factor at $Q^{2}=0.1 \mathrm{GeV}^{2}$ gives

$$
G_{E}^{s}\left(0.1 \mathrm{GeV}^{2}\right)=+0.001 \pm 0.004 \pm 0.004 \text {. }
$$

Following the procedure outlined in Ref. [25], chargesymmetry violations (CSV) of the maximum size estimated theoretically [26] are found to be small with respect to the error on the experimental proton mean-square radius. The small magnitude of the strange form factors, predicted here and in Ref. [5], suggests that the experimental signal may be similar in size to the CSV effects. As the precision in experimental programs is improved, a more careful QCD analysis of CSV effects will be required.

Table I provides a comparison of the present calculation and experiment. The quoted errors on the present prediction are comparable to, or even better than, those anticipated in the forthcoming HAPPEX results.

We thank the Australian Partnership for Advanced Computing (APAC) and the South Australian Partnership for Advanced Computing (SAPAC) for supercomputer support enabling this project. This work is supported by the Australian Research Council and by DOE Contract No. DE-AC05-84ER40150, under which SURA operates Jefferson Laboratory.

[1] K. A. Aniol et al. (HAPPEX Collaboration), Phys. Rev. Lett. 96, 022003 (2006); Phys. Lett. B 635, 275 (2006).

[2] D. S. Armstrong et al. (G0 Collaboration), Phys. Rev. Lett. 95, 092001 (2005).

[3] D. T. Spayde et al. (SAMPLE Collaboration), Phys. Lett. B 583, 79 (2004).

[4] F. E. Maas et al. (A4 Collaboration), Phys. Rev. Lett. 93, 022002 (2004); 94, 152001 (2005).

[5] D. B. Leinweber et al., Phys. Rev. Lett. 94, 212001 (2005).

[6] D. B. Leinweber et al., Eur. Phys. J. A 24S2, 79 (2005).

[7] J. M. Zanotti et al., Phys. Rev. D 65, 074507 (2002).

[8] J. M. Zanotti et al., Phys. Rev. D 71, 034510 (2005).

[9] S. Boinepalli et al., Phys. Lett. B 616, 196 (2005).

[10] R. D. Young et al., Prog. Part. Nucl. Phys. 50, 399 (2003); D. B. Leinweber et al., Phys. Rev. Lett. 92, 242002 (2004).

[11] R. D. Young, D. B. Leinweber, and A. W. Thomas, Phys. Rev. D 71, 014001 (2005).

[12] G. A. Miller et al., Phys. Rep. 194, 1 (1990).

[13] D. B. Leinweber, Phys. Rev. D 53, 5115 (1996).

[14] I. Eschrich et al. (SELEX Collaboration), Phys. Lett. B 522, 233 (2001).

[15] S. Eidelman et al. (Particle Data Group), Phys. Lett. B 592, 1 (2004).

[16] M. Falcioni et al., Nucl. Phys. B251, 624 (1985).

[17] S. O. Bilson-Thompson et al., Ann. Phys. (N.Y.) 304, 1 (2003).

[18] G. Martinelli et al., Nucl. Phys. B358, 212 (1991).

[19] D. B. Leinweber, R. M. Woloshyn, and T. Draper, Phys. Rev. D 43, 1659 (1991).

[20] M. Luscher and P. Weisz, Commun. Math. Phys. 97, 59 (1985); 98, 433 (1985).

[21] M. Gockeler et al. (QCDSF Collaboration), Phys. Rev. D 71, 034508 (2005).

[22] R. D. Young et al., Phys. Rev. D 66, 094507 (2002).

[23] S. J. Dong et al., Phys. Rev. D 58, 074504 (1998); N. Mathur and S. J. Dong, Nucl. Phys. B, Proc. Suppl. 94, 311 (2001).

[24] R. Lewis, W. Wilcox, and R. M. Woloshyn, Phys. Rev. D 67, 013003 (2003).

[25] A. W. Thomas et al., nucl-th/0509082.

[26] G. A. Miller, Phys. Rev. C 57, 1492 (1998).

[27] S. Boinepalli et al., hep-lat/0604022. 